\title{
EVALUACIÓN DE SOSTENIBILIDAD DE DOS SISTEMAS DE PRODUCCIÓN CAPRINO: ESTUDIOS DE CASO EN SISTEMAS DE PRODUCCIÓN PECUARIA EN OCAÑA, NORTE DE SANTANDER
}

\author{
Sustainability assessment of two production systems goat: case studies in \\ livestock production systems in Ocaña, North Santander
}

\section{Artículo de Investigación}

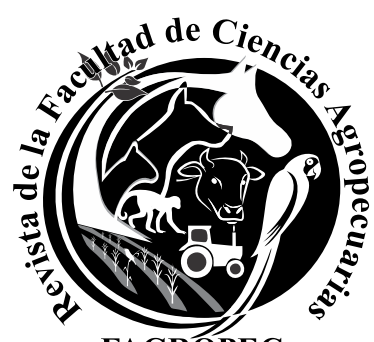

FAGROPEC

Recibido 21 de agosto de 2019. Aceptado 29 de noviembre de 2019.

${ }^{1}$ Docente tiempo completo, Universidad Francisco de Paula Santander, Grupo de investigación GI@DS Y GIPAB.

(iD https://orcid.org/0000-0002-0377-4664

${ }^{2}$ Fundación de Estudios Superiores COMFANORTE, Grupo de investigación GRINFESC y GIPAB.

https://orcid.org/0000-0001-7718-853X

${ }^{3}$ Docente tiempo completo, Universidad Francisco de Paula Santander, Grupo de investigación GI@DS Y GIPAB.

iD https://orcid.org/0000-0003-1971-8365

\section{Como citar:}

HOYOS PATIÑO, J. F., et al. Evaluación de sostenibilidad de dos sistemas de producción caprino: estudios de caso en sistemas de producción pecuaria en Ocaña, Norte de Santander. En: Revista Facultad Ciencias Agropecuarias - FAGROPEC. Universidad de la Amazonia, Florencia - Caquetá. Volumen 11 julio-diciembre, 2019. Pp. 102-118 ISSNRevista en Línea: 2539-178X

*Autor para correspondencia: jfhoyosp@ufpso.edu.co
Johann Fernando Hoyos Patiño ${ }^{1 *}$ MSc. Esp.; Blanca Liliana Velazquez ${ }^{2}$

MSc. Esp. y Daniel Antonio Hernández Villamizar ${ }^{3}$ MSc. Esp.

\section{RESUMEN}

La presente investigación aplicado los criterios e indicadores de sostenibilidad de la metodología "Marco para la Evaluación de Sistemas de Manejo de Recursos Naturales incorporando Indicadores de Sostenibilidad (MESMIS)" en el análisis de dos sistemas de producción comercial de caprinos en el municipio de Ocaña. Evaluando el cumplimiento de las dimensiones económica, ambiental y social. Los indicadores, se unificaron y ponderaron participativamente con los productores. La valoración de los indicadores permitió observar el estado actual de la sostenibilidad, mostrando buen nivel de cumplimento en las tres dimensiones. Igualmente, se revelan puntos a mejorar en la dimensión económica, en donde la carencia de registros contables y financieros no permite la determinación real de los costos de producción. Por otro lado, la falta de registros sanitarios para comercialización, impiden la incursión en nuevos mercados, limitando las potencialidades genéticas y de transformación de productos.

Palabras claves:

MESMIS; Sostenibilidad; Indicador; Caprinos; Ocaña

\section{ABSTRACT}

The present research applied the sustainability criteria and indicators of the methodology "Framework for the Evaluation of Natural Resources Management Systems incorporating Sustainability Indicators (MESMIS)" in the analysis of two commercial goat production systems in the municipality of Ocaña. Evaluating compliance with the economic, environmental and social dimensions The indicators were unified and weighted in a participatory manner with the producers. The assessment of the indicators allowed us to observe the current state of sustainability, showing a good level of compliance in all three dimensions. Likewise, points to be improved are revealed in the economic dimension, where the lack of accounting and financial records does not allow the real determination of production costs. On the other hand, the lack of health records for commercialization, prevent the incursion into new markets, limiting the genetic and transformation potential of products.

Key words:

MESMIS; Sustainability; Indicator; Goats; Ocaña. 


\section{INTRODUCCIÓN}

En la actualidad en el departamento Norte de Santander, el auge de los sistemas de producción caprinos va en aumento, pasando de un número no mayor a 3.000 animales reportado por el Plan de Desarrollo para Norte de Santander 2016-2019 "Un Norte Productivo Para Todos" (PDNS, 20162019) a 18,179 ejemplares referenciados por el Censo Pecuario Nacional - 2018 (ICA, 2018), jugando un papel importante como nueva alternativa en el desarrollo económico del País (Contexto Ganadero, 2013). Por otro lado, el uso técnicas de manejo inapropiado, implementación de sistemas productivos invasivos y la aplicación indiscriminada de productos veterinarios y agroquímicos, genera la perdida de fertilidad del suelo.

De igual manera, las alteraciones en los ecosistemas reflejan un balance desfavorable e inquietante de pérdida de cobertura en bosques naturales para transfórmalas en áreas para la producción pecuaria, proyectando para en área silvo- agrícola 136.047 ha; silvo-pastoril 12.326 ha y producción comercial 1.894 (PDNS, 2016-2019). Esta práctica, se convierte en el principal factor de pérdida de las características del suelo, deteriorando el ecosistema que se debe compensar con la apropiación de técnicas de producción amigables con el ambiente.

Debido a esto, medir la sostenibilidad de los sistemas de producción bajo la metodología MESMIS en sistemas caprinos; se convierte en una alternativa que evitara incurrir en errores del pasado, al momento de implementar prácticas de manejo, procesos de inversión y efectos medio ambientales, que conviertan estos procesos en factor de pobreza para el productor agropecuario. Ante esta situación, se generan algunas interrogantes de investigación.

La metodología MESMIS, propone cinco grupos de atributos para la medir la sostenibilidad, que propician un entorno lógico y completo que hace integral el análisis. El primero, la productividad, capacidad del sistema de producción (SP) para brindar recursos y beneficios; la estabilidad es la característica de mantener en equilibrado dinámico el SP; la adaptabilidad capacidad de encontrar el estado de equilibrio ante cambios a largo plazo; en cuarto lugar, la equidad y por último la autodependencia que conlleva una regulación hacia el exterior del SP. (López, et al, 2000a)

El objeto de esta investigación es analizar la sostenibilidad de dos (2) SP caprinos del municipio de Ocaña. Utilizando la metodología "Marco para la Evaluación de Sistemas de Manejo de Recursos Naturales incorporando Indicadores de Sustentabilidad (MESMIS)", realizando la caracterización de los sistemas y la medición de criterios e indicadores reconocidos por los productores, dentro de las dimensiones de sostenibilidad económica (equilibrio económico), sostenibilidad ambiental (respeto al medio ambiente) y sostenibilidad social (equidad y justicia social) (López, et al, 2000b).

\section{MATERIALES Y MÉTODOS}

La investigación se desarrolló en el municipio de Ocaña, ubicado en la zona Centro Occidental del departamento, y pertenece a la sub-región noroccidental, limita por el Oriente con los municipios de San Calixto, La Playa y Abrego. Por el Norte con los municipios de Teorama, Convención y El Carmen. Por el Sur con el municipio de Ábrego. Por el Occidente. Con los municipios de San Martín 
y Río de Oro (Alcaldía de Ocaña, 2018).

En el contexto Nacional Ocaña hace parte del Norte de Santander, ubicado sobre la cordillera Oriental en una zona completamente montañosa, tiene una extensión aproximada de $627.72 \mathrm{Km} 2$ que representa el 2,76 \% del área total del Departamento, la cabecera municipal se encuentra a una distancia de $203 \mathrm{Km}$. De la capital del Departamento por la vía Ocaña-Cúcuta, además se comunica con el Departamento del Cesar en la vía Rio de Oro-Aguachica, empalmando con la carretera que va a la Costa Atlántica y hacia el centro del país, de igual manera se comunica con la capital del Departamento por la vía Convención - Tibú - Cúcuta (Alcaldía de Ocaña, 2018).

El tipo de investigación aplicada fue descriptiva, reseñando las características de un fenómeno existente, atreves de la recolección de datos, interpretación y análisis en atención al universo real de donde proviene (Arias, 1999); caracterizando dos (2) SP como muestra representativa de procesos de explotación comercial semi-estabulados y estabulado. Dicha selección de SP, se amparado en el tipo de muestra no probabilística por conveniencia (Balestrini, 2006), el cual permite seleccionar la población objeto de estudio, dadas las características de disponibilidad de acceso, colaboración de los productores y presupuesto para la ejecución de la investigación (Bernal, 2006).

\section{Descripción del sistema de producción:}

La metodología utilizada para diagnosticar los SP y desarrollar las socializaciones de seguimiento del proceso participativo, comprendió los siguientes ítem: delimitación de objetivos, aclaración de dudas, análisis de resultados, lluvia de ideas y conclusiones (Cárdenas, 2005).

Para la descripción del SP, se realizó diagnostico con los productores, utilizando tres herramientas participativas descritas por Geilfus en 1997: mapa de la comunidad (adaptado al sistema productivo, que abarca potenciales naturales del SP, dedicación de la tierra, parámetros de calidad de vida), análisis DOFA y matriz de Vester para priorización de problemas (Delgado, 2015).

\section{Selección de los criterios e indicadores}

Como resultado de las entrevistas con los productores, se generó un listado de indicadores potenciales a evaluar; seleccionando los estratégicos. Buscado equilibrio en el número para abracar las dimensiones de la sostenibilidad, cumpliendo con las siguientes características: practicidad en la aplicación, simplicidad de medición, susceptibilidad a los cambios, fácil entendimiento para todas las personas involucradas en el proceso (Glave, 2000).

\section{Medición y seguimiento de los criterios e indicadores}

La evaluación y seguimiento de indicadores, se efectuó para cada dimensión de sostenibilidad. La dimensión social (DS) y la económica (DE), aplicando entrevistas semiestructuradas, talleres y fuentes secundarias. Para la ambiental, con fuentes secundarias, evaluación directa, visita de campo y encuesta. Para analizar los indicadores, los resultados se llevaron a unidades estandarizadas, Según escala recomendada por (López, et al, 2000b) y (Altieri, 2000) en escala sencilla del 0 al 5, se consideró para esta investigación el valor 5 como el más sostenible y 3 como el valor aceptable o rango umbral (Gayoso, 1991).

La consolidación de resultados, integro los aportes de los productores y del equipo investigador, con 
los cuales se construyó un diagrama o mapa de sostenibilidad para cada SP. Se presentan los resultados, usando gráficos en forma de AMIBA o Radial (Astier, et al, 2008), donde el valor obtenido un por cada indicador se representa con un punto y el resultado general con una línea.

Descripción y análisis de los indicadores

La metodología aplicada para la construcción de criterios e indicadores, determino una serie de índices estandarizados para evaluar las tres dimensiones de sostenibilidad: (económica (DE), ambiental (DA) y social (DS)).

Tabla 1: Relación de dimensiones evaluadas, numero de atributos, criterios de diagnóstico e indicadores.

\begin{tabular}{lccc}
\hline \multicolumn{1}{c}{ DIMENSIÓN } & ATRIBUTO & CRITERIO DE DIAGNOSTICO & INDICADOR \\
\hline Dimensión Económica (DE) & 4 & 5 & 13 \\
Dimensión Ambiental (DA) & 3 & 5 & 7 \\
Dimensión Social (DS) & 4 & 7 & 12 \\
\hline & Totales: & 17 & 32 \\
\hline
\end{tabular}

Análisis de la dimensión económica $(D E)$

Para evaluar la viabilidad económica de los SP se eligieron cinco criterios y 13 indicadores:

A- Criterio de Autosuficiencia (CA). Se midió usando tres índices:

A1- Diversidad en la producción. Un sistema es sostenible si la producción tiene variedad de productos: más de 9 productos (5); de 8 a 9 productos (4); de 6 a 7 productos (3); de 4 a 5 productos (2); de 2 a 3 productos (1); inferior a 2 productos. (0).

A2-Necesidad de insumos externos. Un sistema de producción que tenga alta necesidad de insumos externos es inviable en el tiempo: (5): 0 a 10\%; (4): 10 a $20 \%$; (3): 20 a $40 \%$; (2): de 40 a $60 \%$; (1): 60 a $80 \% ;(0): 80$ a $100 \%$.

A3-Nivel de autofinanciamiento. (5) el SP genera todo el capital para soporta la inversión necesaria. (4) 90 a $80 \%$; (3) el SP genera el 80-60\% de capital; (2) 40 a 60\%; (1) 20 a 40\%; (0) el SP genera menos del $20 \%$ del capital.

\section{B-Criterio de Ingreso promedio mensual del trabajador (CIPMT)}

El sistema es sostenible si satisface las necesidades económicas del empleado. Se evalúa en pesos por mes. (5): + de $\$ 781,242$ pesos (salario mínimo año 2018); (4): \$ 700.000- \$ 781,242 pesos; (salario mínimo 2018); (3): \$ 600.000- \$ 699.000; (2): \$ 500.000- \$ 599.000; (1): \$ 400.000- \$ 499.000; (0): $\$ 399.000$

C-Criterio de diversificación de las actividades productivas (CDAP). Se estimó usando seis indicadores.

C1-Diversidad para la venta. Un sistema de producción es sostenible si puede comercializarse más 
de 1 producto o reemplazar la entrada principal por otro. 6 o más productos $(5) ; 5$ productos (4); 4 productos $(3) ; 3$ productos $(2) ; 2$ productos $(1) ; 1$ producto $(0)$.

C2-Canales de comercialización. Variedad en los mercados minimiza el riesgo económico. 6 o más canales (5); 5 canales (4); 4 canales ( 3$) ; 3$ canales (2); 2 canales (1); 1 canal ( 0$)$.

C3- Proceso de sacrificio de los animales: La región posee un plantel de sacrificio debidamente avalado y certificado por la autoridad competente, para garantizar la comercialización en nuevos mercados. (5) Existe planta de sacrificio regional; (4) sacrifica dentro del predio, cumpliendo con la normativa mínima exigida por la autoridad competente; (3) venta de animales en pie.

C4-Venta directa o intermediarios: (5) 80-100\% de la producción comercialización propia; (3) 40$60 \%$ de la producción venta propia; (0) toda la producción vendida a intermediarios.

C5-Cumplimiento de normativa sanitaria para venta de productos. Los productos elaborados en el sistema de producción cuentan, con los registros sanitarios o permisos para su comercialización en el mercado: (5) todos los productos poseen licencias sanitarias; (4) parte de los productos tienen registros sanitarios; (3) no tiene registro sanitario, pero cumple con los procesos sanitarios y de estandarización para su elaboración; (2) no realiza procesos de transformación de productos.

C6- Implementación de procesos de transformación de productos para diversificar mercados. (5) $80-100 \%$ de la producción se transforma para la venta; (4) 40-60\% de la producción se transforma para la venta; (3) NO se realizan proceso de trasformación venta directa de los productos.

\section{D-Criterio promedio Ingreso neto mensual del sistema de producción (CPIM).}

Aunque este criterio depende de la capacidad instalada del SP y el número de animales en producción, se da un valor promedio de ingresos, según el salario mínimo vigente para el año de estudio 2018 de \$ 781,242 pesos. (5) más de 5 SMMV; (4) entre 4 a 5 SMMV; (3) entre 3 a 4 SMMV; (2) menos de 2 a 3 SMMV (SP de producción recién establecido o en proceso de crecimiento); (1) 1 a 2 SMMV; (0) 1 SMMV (SP de producción recién establecido o en proceso de crecimiento).

E-Criterio registros contables y financieros (CRCF). Se estimó con un indicador.

E1-Registros contables y financieros (determinación real de costos de producción). (5) SI implementa registros contables y financieros; (3) registros contables y financieros a medias; (0) NO llevan programas de registros contables y financieros.

F- Criterio de evolución del empleo (CEE). Se estimó con dos indicadores.

F1-Empleos generados de tiempo completo para el manejo del sistema de producción. (5): uno a más trabajadores con dedicación exclusivamente para el manejo del SP; (3): un trabajador con porcentaje de la labor diaria dedicada al sistema y el resto de las labores pagadas por jornal.

F2-Contratación bajo ley laboral. (5) cumple con toda la normativa legal de contratación; (3) 
contratación únicamente del administrador cumpliendo con la normativa legal y contratación de jornales; (2) toda la contratación exclusiva por jornal diario; (0) ocasionales por cumplimento de tarea.

Determinación dimensión económica:

Por las características del proceso productivo se priorizaron los siguientes indicadores: Diversidad en la producción; Nivel de autofinanciamiento; Diversidad para la venta; Proceso de sacrificio de los animales; Cumplimiento de normativa sanitaria para venta de productos y al criterio Registros contables y financieros. A estos índices y un criterio en la evaluación, se les asigno el doble del valor que al resto. La calificación de la Dimensión Económica (DE), se estimó con la suma de los indicadores multiplicado por su valor, con la siguiente formula:

$$
D E=\frac{\left[\frac{[(2 A 1)+A 2+(2 A 3)]}{5}+B+\frac{[(2 C 1)+C 2+C 3+(2 C 4)+(2 C 5)+C 6]}{9}+D+(2 E)+\frac{F 1+F 2}{2}\right]}{7}
$$

Donde:

DE: Dimensión Económica: A: Criterio de Autosuficiencia, A1, A2, A3: Indicadores para evaluar este criterio. B: Criterio de Ingreso promedio mensual del trabajador, C: Criterio de diversificación de las actividades productivas. C1, C2, C3, C4, C5, C6: Indicadores para evaluar este criterio. D: Criterio promedio Ingreso neto mensual del sistema de producción. E: Criterio registros contables y financieros. F: Criterio de evolución del empleo. F1, F2: Indicadores para evaluar este criterio.

\section{Análisis de la dimensión ambiental (DA)}

La dimensión ambiental (DA), se evaluó a través de cinco índices:

\section{A-Criterio de Conservación del suelo (CCS).}

Un sistema es sostenible si las prácticas protegen o mejoran la vida del suelo.

A1- Cobertura vegetal. Provee al suelo protección contra riesgo de erosión y agentes climáticos. (5): $100 \%$ de cobertura; (4): 99 a $81 \%$; (3): 80 a $61 \%$; (2): 60 a $41 \%$;(1): 40 a $21 \%$; (0): <20\%.

\section{B-Criterio de Diversidad de especies y/o géneros (CDEG)}

B1- Diversificación de cultivos (pasto de corte y forrajeras) para la alimentación animal. (5): Completamente diversificado, asociando cultivos y vegetación natural; (4): Diversificación alta de cultivos, con asocio medio entre ellos; (3): mediana diversidad, con bajo nivel de asocio entre ellos; (2): Poca diversidad de cultivos, sin asocio; (1): Monocultivo. (0): Alimenta directamente del bosque.

\section{C-Criterio de Probabilidad de erosión (CPE).}

Un sistema es sostenible si logra minimizar la pérdida de suelo debido a la erosión hídrica.

C1- Pendiente predominante. (5): Topografía plana; (4): del 0 al $5 \%$; (3): del 5 al $15 \%$; (2): del 15 al 
$30 \%$; (1): del 30 al $45 \%$; (0): mayor al $45 \%$.

C2-Orientación de surcos. (5): Uso de terrazas o curvas de nivel (4): no se hace necesario por el tipo de topografía; (3): Siembra perpendicular a la pendiente; (2): Sembrados orientados a $60^{\circ}$ en relación a la pendiente; (1): Surcos orientados $30^{\circ}$ en relación a la pendiente; (0): Siembra paralela a la pendiente.

\section{D- Criterio de Renovación de la Biodiversidad (CRB).}

D1- Biodiversidad temporal. Renueva o resiembra los pastos cultivados en los potreros. Esto incrementa la diversidad en el tiempo. (5): Renueva o resiembra cada año. Descansando un año el potrero o usa abonos verdes; (4): Renueva o resiembra anualmente. Sin descaso para el suelo; (3): Renueva o resiembra cada 2 o 3 años; (2): Renueva o resiembra eventualmente; (1): Renueva o resiembra cada 4 o 5 años; (0): No realiza renovación o resiembra

D2- Zonas de conservación: Las áreas de conservación están compuestas por bosques, nacimientos, orillas de ríos y cañadas, donde NO se llevan a cabo labores agrícolas ni pecuarias y están delimitadas. (5) protege rigurosamente con cercados y siembra de especies vegetales que favorezcan el recurso; (3) protege con cercados, pero NO siembra de especies vegetales que favorezcan el recurso; (0) No tiene ningún área de conservación.

\section{E- Criterio de Conservación de recursos (CCR) (aprovechamiento materia orgánica)}

E1- Tratamiento de Excretas. (5) Recolección en tanque estercolero y composta antes de aplicar; (4) Recolección en un lugar adecuado y después de un tiempo aplicación directa en potreros; (3) recolecta y aplica directamente a los potreros; (0) No tratar y verter directamente al medio ambiente.

\section{Determinación dimensión Ambiental (DA):}

Por las características del proceso productivo se priorizaron los siguientes indicadores: Diversificación de cultivos; Pendiente predominante y a los criterios de Renovación de la biodiversidad y conservación de recursos. A estos índices en la evaluación, se les asigno el doble del valor que al resto. La calificación de la Dimensión Ambiental (DA), se estimó con la suma de los indicadores multiplicado por su valor, con la siguiente formula:

$$
D A=\frac{\left[A+(2 B)+\frac{[(2 C 1)+C 2]}{3}+\frac{[D 1+(2 D 2)]}{3}+2 E\right.}{7}
$$

Donde:

DA: Dimensión Ambiental., A: Criterio de conservación del suelo. B: Criterio de diversidad de especies y/o géneros. C: Criterio de probabilidad de erosión. C1, C2: Indicadores para evaluar este criterio. D: Criterio de renovación de la Biodiversidad. D1, D2: Indicadores para evaluar este criterio. E: Criterio de conservación de recursos (aprovechamiento de materia orgánica) 


\section{Análisis de la dimensión social (DS):}

\section{A-Criterio de Cumplimiento de las necesidades básicas del empleado (CCNBE).}

A1- Vivienda. En material y terminada (obra blanca) (5); En material terminada. Buena (4); En material terminada. (Obra negra) (3); Regular estado. En construcción o descuidada (2); Sin terminar, descuidada, piso en tierra (1); rancho de tabla, madera o cartón (0).

A2- Acceso a la educación. Educación superior (5); Cursos de capacitación (4); Escuela secundaria (3); Escuela primaria y secundaria con restricciones (2); Escuela primaria (1); Sin acceso a la educación $(0)$.

A3- Cobertura de salud: Centro de salud con médico permanente con infraestructura adecuada (5); Centro de salud con médico permanente e infraestructura regularmente adecuada (4); Centro de salud con personal temporal regularmente dotado (3); Centro de salud mal equipado y personal temporal(2); Centro de salud sin personal capacitado y sin equipado (1); Sin centro de salud(0).

A4- Servicios básicos. (5): Agua, luz, cobertura señal de celular e internet; (4): Agua, luz y cobertura señal de celular; (3): agua y luz; (2): luz y agua de pozo; (1): Sin luz y agua de pozo cercano; (0): Sin luz y sin fuentes de agua cercanas.

\section{B- Criterio de Gusto por el sistema de producción (CGSP).}

Agrado del productor y del empleado relacionado con el nivel de afinidad con el SP. (5): El sistema de producción es muy a fin a sus gustos. No trabajaría en otra actividad, aunque le reporte más ingresos; (4): El sistema de producción es muy a fin a sus gustos; (3): Está contento, pero anteriormente le iba mejor; (2): No está del todo contento. Lo hace porque es lo único que sabe hacer; (1): Poco contento con esta forma de vida. Prefiere vivir en la ciudad y ocuparse en otro empleo; (0): Está desconforme con la vida que lleva.

\section{C-Criterio de Integración social (CIS).}

C1- Miembro de asociaciones. Se evaluará la participación en asociaciones gremiales. En la escala de valoración se da como referente que lo ideal es la participación en la totalidad de las asociaciones existentes en la región. (5) Participa en todas las asociaciones; (3) participación en una asociación; (0) no participa en ninguna asociación.

\section{D- Criterio de Consciencia Ecológica (CCE).}

La conciencia ecológica es fundamental para la toma de decisiones referentes a la conservación. (5): Entiende la ecología desde una visión general, más amplia que su sistema de producción y conociendo sus fundamentos; (4): Tiene conocimiento ecológico desde su cotidianidad. Sin el no uso de agroquímicos y prácticas de conservación; (3): Visión parcial de la ecología. Cree que algunas prácticas perjudican el medio ambiente; (2): No tienen conocimiento ecológico. Pero aplica prácticas con bajos insumos agrícolas; (1): No tiene conocimiento ecológico ni denota consecuencias que ocasionarían algunas prácticas, utilizando altos insumos; (0): Sin conciencia ecológica. 


\section{E-Criterio de Organización (CO)}

E1- Apoyo Institucional (Alcaldías, secretarias de gobierno, empresa pública y/o privada e instituciones educativas). (5) apoyo de las instituciones públicas/privadas existentes en la región para el desarrollo del SP; (3) apoyo parcial apoyo de las instituciones públicas/privadas; (0) No existe apoyo de las instituciones públicas/privadas.

\section{F-Criterio de Capacidad de cambio e innovación (CCCI)}

F1- Consciencia de cambio e aplicación de nuevas tecnologías (innovación) en los últimos 5 años. Tecnologías y procesos innovadoras implementados en los SP como preparación del suelo, semillas certificadas, maquinaria, riegos, uso de biotecnologías reproductivas, manejo de software, reproductores puros entre otras. (5) más de 6; (4) 5 a 6 ; (3) 3 a 4; (2) 2 ; (1) 1 ; (0) 0

F2- Generación de conocimiento. Participa en procesos de capacitación en el área y generando conocimiento a través de la entrevista y visita a las explotaciones. Es importante que el SP se actualice, por lo tanto, se requiere mínimo 10 capacitaciones al año. (5) más de 10; (4) 7 a 10; (3) 5 a $7 ;(2) 3$ a $5 ;(1) 1$ a $3 ;(0) 0$

F3- Valoración del Bienestar animal. (5) valoración y medición de bienestar animal (BA) aplicando protocolos adaptados al SP; (3) es importante el BA en el sistema de producción, pero no se tiene establecido un proceso de valoración y medición; (0) no es relevante la valoración y medición de BA.

\section{G-Criterio de Grado de democratización (CGD)}

G1- Mecanismos de distribución del poder en la toma de decisiones. (5) toma de decisiones prospectivas del SP en conjunto entre el propietario, administrador y asesor técnico; (3) toma de decisiones en conjunto entre el propietario y administrador; (0) toma de decisiones únicamente por el propietario.

\section{Determinación dimensión Social:}

Por las características del proceso productivo se priorizaron los siguientes indicadores: Consciencia de cambio e aplicación de nuevas tecnologías (innovación) en los últimos 5 años y Generación de conocimientos. A estos índices en la evaluación, se les asigno el doble del valor que al resto. La calificación de la Dimensión Social (DS), se estimó con la suma de los indicadores multiplicado por su valor, con la siguiente formula:

$$
D S=\frac{\left[\frac{A 1+(A 2)+A 3+A 4}{4}+B+C+D+E+\frac{[F 1+(2 F 2)+(2 F 3)]}{4}+G\right]}{7}
$$

Donde:

DS: Dimensión social: A: Criterio de cumplimiento de las necesidades básicas del empleado. A1, A2, A3, A4: Indicadores para evaluar este criterio. B: Criterio de gusto por el sistema de producción. C: 
Criterio de integración social. D: Criterio de consciencia Ecológica. E: Criterio de organización F: Criterio de capacidad de cambio e innovación. F1, F2, F3: Indicadores para evaluar este criterio. G: Criterio de grado de democratización.

\section{Índice de sostenibilidad general (ISG)}

Se determinó un valor mínimo o de umbral que debe lograr el índice de sostenibilidad general (ISG) (Sarandón, 2009), para estimar que un SP es sostenible debe promediar un valor igual o mayor al valor medio de escala 3 en una valoración de 0 a 5 . Por otro lado, ninguna de las tres dimensiones debe tener un valor menor a 2 .

$$
I S G=\frac{D E+D A+D S}{3}
$$

Donde:

ISG: Índice de sostenibilidad general. DE: Dimensión económica. DA: Dimensión Ambiental. DS: Dimensión Social.

\section{RESULTADOS Y DISCUSIÓN}

Usar criterios e indicadores de medición permite detectar, a pesar de la semejanza entre los sistemas de producción, una variación de la sostenibilidad en las tres dimensiones. El Indice de sostenibilidad general fue (ISG: 4,02) promediado entre los dos SP, superando el valor medio para aprobar la evaluación (Tabla 2). Obteniendo como promedio de la dimensión económica $(3,98)$; par la dimensión ambiental $(3,95)$ y para la dimensión social $(4,12)$ Figura 1.

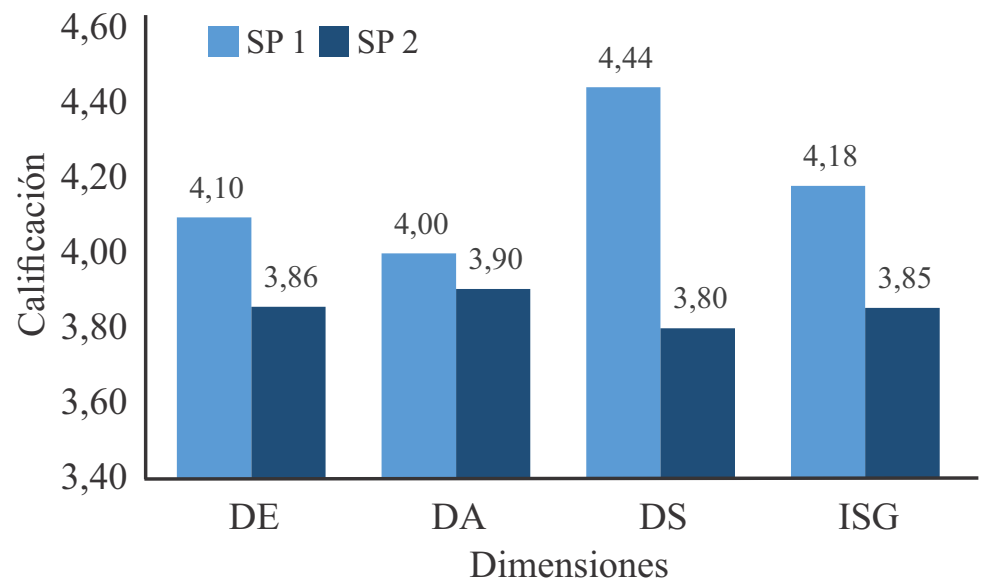

Figura 1: Valores de la dimensión económica (DE), dimensión Ambiental (DA) y dimensión social (DS) de los los dos sistemas de producción del municipo de Ocaña. SP 1 Caprino Semiestabulado; SP 2 Caprino Estabulado; ISG: Índice de sostenibilidad general.

Los criterios e indicadores de la dimensión económica (DE) (Tabla 2) reflejan puntos a mejorar en el criterio de autosuficiencia (CA) para el SP2 en relación al indicador necesidad de insumos externos. Por otro lado, se detectan falencias en el criterio registros contables y financieros (CRCF) para los 
dos SP en el indicador registros contables y financieros realizados a medias. Entre las fortalezas de esta dimensión, resalta el criterio de Ingreso promedio mensual del trabajador (CIPMT), criterio promedio Ingreso neto mensual del sistema de producción (CPIM) y criterio de evolución del empleo (CEE).

Como resultado de la valoración de criterios e indicadores de la dimensión ambiental (DA) (Tabla 2), el SP2 presenta debilidad en el criterio de probabilidad de erosión (CPE) en el indicador de pendiente predominante debido al alto porcentaje de pendiente donde se desarrollan los cultivos. Entre las fortalezas encontradas en esta dimensión para los dos SP, se encuentra el criterio de conservación del suelo (CCS) resaltando el elevado manejo de cobertura vegetal en todo el predio.

Los criterios e indicadores de la dimensión social (DS) (Tabla 2), presentan puntos a débiles para el $\mathrm{SP} 2$ en el criterio de organización $(\mathrm{CO})$ en el indicador apoyo parcial apoyo de las instituciones públicas/privadas y en el criterio de grado de democratización (CGD) en el indicador de toma de decisiones en conjunto entre el propietario y administrador. Como punto a mejorar para los dos SP, resalta el criterio de Integración social (CIS), debido a la participación en una sola asociación del sector.

\begin{tabular}{lcc}
\hline & SP 1 & SP 2 \\
\hline CA & 4,00 & 3,67 \\
CIPMT & 5,00 & 4,00 \\
CDAP & 4,67 & 4,33 \\
CPIM & 4,00 & 5,00 \\
CRCF & 3,00 & 3,00 \\
CEE & 5,00 & 4,00 \\
DE & $\mathbf{4 , 1 0}$ & $\mathbf{3 , 8 6}$ \\
CCS & 4,00 & 5,00 \\
CDEG & 4,00 & 4,00 \\
CPE & 4,00 & 2,33 \\
CRB & 4,00 & 4,00 \\
CCR & 4,00 & 4,00 \\
DA & $\mathbf{4 , 0 0}$ & $\mathbf{3 , 9 0}$ \\
CCNBE & 4,50 & 4,00 \\
CGSP & 5,00 & 5,00 \\
CIS & 3,00 & 3,00 \\
CCE & 4,00 & 4,00 \\
CO & 5,00 & 3,00 \\
CCCI & 4,60 & 4,60 \\
CGD & 5,00 & 3,00 \\
DS & $\mathbf{4 , 4 4}$ & $\mathbf{3 , 8 0}$ \\
ISG & $\mathbf{4 , 1 8}$ & $\mathbf{3 , 8 5}$ \\
Susten & SI & SI \\
\hline & & \\
&
\end{tabular}

Tabla 2. Valores de los criterios en los dos sistemas de producción del municipio de Ocaña. (DE) Dimensión Económica; (CA) Autosuficiencia; (CINM) Ingreso neto mensual por grupo (Eficiencia y Rentabilidad); (CDAP) Diversificación de las actividades productivas; (CPIN) Promedio Ingreso neto mensual del sistema de producción; (CRCF) Registros contables y financieros; (CEG) Evolución del empleo; (DA) Dimensión Ambiental; (CCVS) Conservación del suelo (eficiencia); (CDTE) Diversidad de especies y/o géneros; (CRE) Riesgo de erosión (conservación de recursos); (CMBD) Manejo de la biodiversidad; (CAU) Autosuficiencia; (DS) Dimensión Social; (CSNB) Satisfacción de las necesidades básicas (calidad de vida) del empleado; (CASP) Aceptabilidad del sistema de producción (calidad de vida); (CIS) Integración social (organización); (CCE) Conocimiento y conciencia Ecológica (control); (CO) Organización; (CCCI) Capacidad de cambio e innovación; (CGD) Grado de democratización. 
Dentro de las fortalezas de la dimensión social para los SP se encuentra el criterio de gusto por el sistema de producción (CGSP) resaltando el gran gusto del propietario y trabajadores por la actividad económica realizada y en el criterio de capacidad de cambio e innovación (CCCI) en los indicadores consciencia de cambio e aplicación de nuevas tecnologías, participación en procesos de capacitación y valoración del bienestar animal. Por otro lado, los SP muestran gran compromiso en capacidad y generación de conocimientos, apoyando los procesos de formación de las diferentes universidades e instituciones académicas de las regiones, demostrando así la necesidad del apoyo técnico para la toma de decisiones y mejoramiento constante del sistema.

La comparación de los criterios de los dos sistemas de producción usando un diagrama tipo ameba, permite identificar diferencias en las dimensiones de la sostenibilidad (Figura 2). E1 SP 1 presentado mejor desempeño en los criterios evaluados, con menos puntos a mejorar que el SP 2. Algunos de los aspectos analizados presentaron calificaciones de cinco, y puntos a mejorar en las tres dimensiones para el SP2.

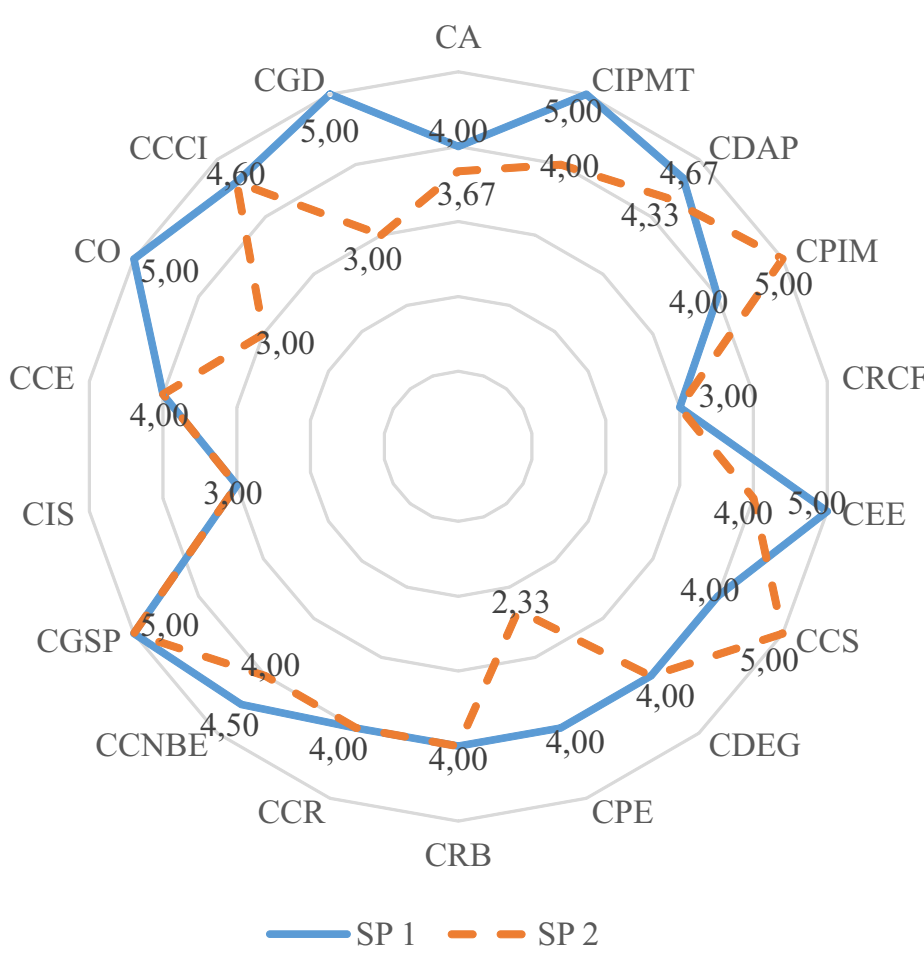

Figura 2. Representación gráfica en un diseño tipo ameba, de los criterios de sostenibilidad en dos sistemas de producción del area metropolitana de la Ciudad de Cucuta y el municipo de Ocaña. El límite exterior representa el valor máximo de sostenibilidad y el intermedio el valor medio o umbral. Línea continua SP número 1; línea punteada SP número 2. sistemas de producción del area metropolitana de la Ciudad de Cucuta y el municipo de Ocaña. (DE) Dimensión Economica; (CA) Autosuficiencia; (CINM) Ingreso neto mensual por grupo (Eficiencia y Rentabildiad); (CDAP) Diversificación de las actividades productivas; (CPIN) Promedio Ingreso neto mensual del sistema de producción; (CRCF) Registros contables y financieros; (CEG) Evolución del empleo; (DA) Dimensión Ambiental; (CCVS) Conservacion del suelo (eficiencia); (CDTE) Diversidad de especies y/o generos; (CRE) Riesgo de erosión (conservación de recursos); (CMBD) Manejo de la biodiversidad; (CAU) Autosuficiencia; (DS) Dimensión Social; (CSNB) Satisfacción de las necesidades basicas (calidad de vida) del empleado; (CASP) Aceptabilidad del sistema de produccion (calidad de vida); (CIS) Integración social (organización); (CCE) Conocimiento y conciencia Ecologica (control); (CO) Organización; (CCCI) Capacidad de cambio e innovación; (CGD) Grado de democratización. 
Los resultados muestran que los dos SP estudios presentan una sostenibilidad alta en algunos de los criterios e indicadores analizados. Es decir, que no están lejos del ideal, especialmente en la dimensión ambiental.

En la dimensión económica se encuentran aspectos a mejorar, en los criterios eficiencia y determinación real de la rentabilidad, factores de gran relevancia al momento de caracterizar SP dedicados a la explotación comercial. Esto debido a la falta de registros financieros y contables, características particulares del proceso productivo, capacidad instalada y demanda del mercado. Sin embargo, esto puede mejorar a través de la implementación de proceso financieros que determinen la utilidad real de los sistemas, la consecución de licencias sanitarias INVIMA para los productos transformados. Con esto se lograría, la incursión en nuevos mercados, favoreciendo la potencialidad actual de estandarización en la elaboración de productos y recurso de alta genético de las explotaciones, mejorando la competitividad.

La sostenibilidad ambiental, compensa parcialmente los valores obtenidos en la dimensión económica y social. La capacidad instalada de los SP permite la contratación exclusiva de mano de obra para el manejo de los animales, donde la mano de obra contratada es exclusiva para la producción de animales de alta genética y líneas comerciales, facilitando en un futuro la determinación de costos de producción y rentabilidad, como lo proyecta Delgado, et al (2010).

Esta evaluación mostro una dependencia alta de las dimensiones estudiadas. La sostenibilidad ambiental de los SP, estuvo claramente limitada por parámetros sociales y económicos. Al analizar el diagrama en tela de araña (Figura 2) se muestra la asociación entre la conservación de los recursos (dimensión ambiental) y el entendimiento ecológico de los productores (dimensión social).

En el SP 5 los altos valores de la dimensión ambiental se corresponden con valores superiores en la dimensión económica. Esto refuerza la relación existente entre la conciencia ambiental del productor y la administración de los recursos para la conservación de la biodiversidad (Garzón y López, 2017). El proceso conciencia ambiental, la inquietud por recuperar y conservar la biodiversidad del ecosistema, representan emprendimientos eco-innovadores que mejora la calidad de vida del productor y los empleados, generando nuevas entradas para el sistema (Andersen, 2008 citado por Arroyave, et al., 2019).

Las diferencias en la valoración de los SP, al ser analizados desde diferentes dimensiones (económico, ambiental y social) de manera parcial, confirma que un manejo económico apropiado, no obligatoriamente es el más sostenible, el análisis costo-beneficio, instrumento económico, desconoce los costos ocultos producto del proceso de producción, el desgaste de los recursos y su deterioro. Por tal razón, decisiones económicamente razonables pueden ser, a su vez, ambientalmente insostenibles (López-Ridaura, S; Astier, M \& Masera, O. 2000a).

Los resultados obtenidos en esta investigación, confirman el beneficio del empleo del enfoque sistémico y adaptado para la caracterización de los SP, con un enfoque de criterios e indicadores para abordar las tres dimensiones de la sostenibilidad Garzón B D C y López MA D. (2017). Con el uso de esta metodología se posibilita el análisis de varios objetivos al mismo tiempo. El resultado no se mide por un solo resultado, sino promediando varias posibilidades, que brindan los criterios e indicadores 
(Sarandón, et al., 2002). Para este estudio, el peso de los criterios e indicadores surge participativamente, como se han realizado varios estudios (Sarandón, et al. 2003; Geilfus F. 2009; Silva S L. 2014; Salazar C O L. (2015); Garzón B D C y López M A D. (2017).

Con el análisis de cada uno de los factores del sistema de producción se evidencia que cada componente aporta a la sostenibilidad, como la reducción de costos de producción usando racionalmente el agua, producción de alimento e implementación de bancos de proteína, aprovechamiento de subproductos y la elaboración de abonos orgánicos, ayudando a la producción de valor económico (Arroyave y Marulanda, 2019).

Por otro lado, los resultados de esta valoración pueden variar, si el peso asignado a los diferentes criterios e indicadores, hubiese sido diferente. Pero se incluye la participación de los productores en esta decisión, para arrojar resultados acordes a la realidad del proceso productivo, como sugiere Delgado, A. et al. (2007) y Niño C D. (2012).

Tomando como punto de partida el concepto de sostenibilidad los SP evaluados deben buscan dicho equilibrio generando interacción entre las dimensiones económica, ambiental y social, enfocándose en el cumplimiento de las características descritas por Dyllick y Hockerts (2002) citado por Arroyave et al (2019): Para ser sostenible económicamente, se debe garantizar efectivo disponible para mantener la liquidez y aumentar el rendimiento financiero. Para lograr ser sostenible ecológicamente, en relación al uso de recursos naturales su consumo debe ser inferior al ritmo de renovación del ecosistema y sostenible socialmente, brindando estabilidad y desarrollo a las comunidades donde tienen injerencia.

\section{CONCLUSIONES}

Durante el desarrollo de la investigación se identificaron dos tipos de producción de caprinos; uno semiestabulado realizado por los SP 1 y otro de tipo estabulada presente en el SP 2. El semiestabulado, caracterizada por la implementación de pastoreos durante ciertas horas del día, suplementación de concentrados y sales mineralizadas en los apriscos; y el estabulado, basada en el confinamiento total de los animales en el aprisco, suministrando heno, concentrados y sales mineralizadas.

Los SP objeto de estudio, presentan en promedio una extensión entre 6 a 8 hectáreas, en las cuales se tiene distribuido los cultivos para la alimentación de los rebaños y las instalaciones necesarias para el proceso productivo. El tipo de forraje predominante se adapta a las condiciones climáticas de la zona y presenta buen aforo por $\mathrm{m}^{2}$, debido a las condiciones de manejo aplicadas; las áreas productivas están delimitadas con cercas (tradicional o eléctrica); el cuidado y conservación de las nacientes de agua es prioridad.

El proceso de selección de indicadores participativamente con los propietarios de los SP, brinda la certeza de su idoneidad y facilidad de aplicación, permitiendo así, evaluar cada dimensión objetivamente. Con la evaluación, constató que los dos SP caprinos valorados, se pueden considerar como sostenibles, cumpliendo con el parámetro o umbral de valoración para tal fin, calificación mayor a tres 3,0 . 
De manera general, es posible concluir que el uso de criterios e indicadores para determinar sostenibilidad en los SP es un método efectivo, que se fortalece con la participación de los productores, para evaluar la realidad del contexto partiendo de las debilidades y fortalezas. Sin embargo, el desarrollo de la actividad caprina en el en el municipio de Ocaña, surge como alternativa económica creciente.

\section{RECOMENDACIONES}

- Para un mejor aprovechamiento de la aplicación de la metodología MESMIS, se sugiere extender el estudio para establecer una ruta que permita mejorar la sostenibilidad de los SP caprinos de la región.

- Implementar estrategias de seguimiento y mejoramiento constante en la medición de sostenibilidad en los sistemas estudiados.

- Implementar registros financieros que permitan determinar los costos reales de producción y rentabilidad de los sistemas.

- Fomentar los procesos de aprovechamiento de subproductos y transformación de residuos para la optimización de los ciclos energéticos.

\section{LITERATURA CITADA}

ALTIERI, M.; NICHOLLS, C. Teoría y práctica para una agricultura sostenible. Serie de Textos Básicos para la Formación Ambiental. Programa de las Naciones Unidas para el Medio Ambiente. México: Red de Formación ambiental para América Latina y el Caribe, 2000.

ARIAS, Fidias; FIDIAS, G. El Proyecto de Investigación: Guía para su elaboración. 3ra. Edición, Editorial Episteme. Caracas, 1999.

ANDERSEN, Maj Munch. Eco-innovation-towards a taxonomy and a theory. En 25th celebration DRUID conference. 2008.

ARROYAVE-PUERTA, Adriana María; MARULANDA-VALENCIA, Flor Ángela. Ecoemprendimiento, sostenibilidad y generación de valor. Revista Escuela de Administración de Negocios, 2019, no 87. DOI: https://doi.org/10.21158/01208160.n87.2019.2411

ASTIER, Marta; MASERA, Omar R.; GALVÁN-MIYOSHI, Yankuic. Evaluación de sustentabilidad: un enfoque dinámico y multidimensional. Valencia: SEA E, 2008 . https://www.researchgate.net/publication/319325816_Evaluacion_de_sustentabilidad_Un_enfoque_dinam ico_y_multidimensional

BALLESTRINI, Mirian. Cómo se elabora el proyecto de investigación. Caracas. BL Consultores Asociados, Servicio Editorial, 2001.

BERNAL TORRES, Cesar Augusto. Metodología de la Investigación. $2^{\text {a }}$ Edición. Editorial Pearson. México. 2006.

CARDENAS GGI, GIRALDO GH; IDÁRRAGA, Q. A.; VÁSQUEZ, G. L. N. Desarrollo y validación de metodologías para evaluar con indicadores la sustentabilidad de sistemas productivos campesinos de la 
asociación de cafeticultores orgánicos de Colombia-ACOC. Disponible en Disponible en http://www. javeriana. edu.co/ear/m_des_rur/documents/Cardenas-ponencia.pdf Publicado en, 2006.

SEGURA, O. La ganadería ovina vive su mejor momento en Colombia. Contexto Ganadero. [En línea]. http://www. contextoganadero. com/reportaje/la-ganaderia-ovinavive-su-mejor-momento-en-colombia, 2013.

DELGADO, A., ARMAS, W., D'AUBETERRE, R., HERNÁNDEZ, C., \& ARAQUE, C. Sostenibilidad del sistema de producción Capra hircus-Aloe vera en el semiárido de Cauderales (estado Lara, Venezuela). A groalimentaria, 2010 , vol. 16, no 31, p. $49-63$. D isponible en: http://ve.scielo.org/scielo.php?script=sci_arttext\&pid=S1316-03542010000200004

DELGADO, A., ARMAS, W., D'AUBETERRE, R. \& ARAQUE, C. Evaluación de la sostenibilidad de un sistema de producción caprino, utilizando indicadores. gaceta de Ciencias Veterinarias, 2007, vol. 13, no 1, p. 45-52. ISSN 1690-8414, Vol 13 n$^{\circ} 1$ [citado 03 junio 2015]. Disponible en: https://goo.gl/nqiKNk

DYLLICK, Thomas; HOCKERTS, Kai. Beyond the business case for corporate sustainability. Business strategy and the environment, 2002, vol. 11, no 2, p. 130-141.

GARZÓN BRAVO, Daysy Catherine; LÓPEZ MONCAYO, Ángela Dajhana. Evaluación de la sostenibilidad de los sistemas de producción implementados por la Asociación Agropecuaria de Timbío (Cauca). 2017. Recuperado de: https://goo.gl/JqAsGf

Gayoso J,Iroumé A.(1991).Metodología para estimar la fragilidad de terrenos forestales. Medio Ambiente 11(2): 13-24.

GEILFUS, Frans. 80 [ochenta] herramientas para el desarrollo participativo: diagnóstico, planificación, monitoreo, evaluación. PROCHALATE, 1998.

GLAVE, Manuel; ESCOBAL, Javier. Indicadores de sostenibilidad para la agricultura andina. Boletín A g r o e c ológ i co. XI.67., 2000, vol. 20, p. 32 . D is ponible e n: http://bibvirtual.ucla.edu.ve/db/psm_ucla/edocs/gcv/Vol13Nro1/articulo7.pdf

ICA. Censo Pecuario Nacional. 2016.

LÓPEZ-RIDAURA, Santiago; MASERA, Omar; ASTIER, Marta. Evaluando la sostenibilidad de los sistemas agrícolas integrados: el marco MESMIS. Revista Leisa de Agroecología, 2001, vol. 16, p. 25-27.

MASERA, O.; ASTIER, M.; LÓPEZ-RIDAURA, S. Sustentabilidad y manejo de los recursos naturales. El marco de evaluación MESMIS. Grupo Interdisciplinario de Tecnología Rural Apropiada (GIRAAC). 1999.

NIÑO CARREÑO, Delia. Caracterización de la producción de caprinos bajo sistemas silvopastoriles en la vereda la jabonera del municipio de soata-Boyacá. 2013. Trabajo de grado como requisito para optar al título de Especialista en Nutrición Animal Sostenible. Universidad nacional abierta y a distancia "UNAD" facultad de ciencias agropecuarias y medio ambiente. Duitama. https://goo.gl/jv8Qjw

GOBERNACIÓN DE NORTE DE SANTANDER. Plan de desarrollo para Norte de Santander 2016-2019. Recuperado de http://www. sednortedesantander. gov. co/sitio/images/documentos/informesdelsector/PDD\% 20NDS, 2016, vol. 20, p. 2016-2019. 
SALAZAR CÁRDENAS, Olga Lucía. Evaluación de la implementación de Buenas Prácticas Pecuarias en la producción de ovinos y caprinos en la zona metropolitana de los municipios de Bucaramanga y Lebrija. 2015. (Tesis de Pregrado). Universidad de Manizales. Manizales, Colombia

SARANDÓN, Santiago J., et al. Evaluación de la sustentabilidad del manejo de suelos en agroecosistemas de la provincia de La Pampa, Argentina, mediante el uso de indicadores. Revista brasileira de agroecologia, 2006, vol. 1, no 1 .

SARANDÓN, Santiago J., et al. Evaluación de la sustentabilidad de sistemas agrícolas de fincas en Misiones, Argentina, mediante el uso de indicadores. Agroecología, 2006, vol. 1, p. 19-28.

SARANDÓN, Santiago J. El desarrollo y uso de indicadores para evaluar la sustentabilidad de los agroecosistemas. Agroecología: El camino para una agricultura sustentable, 2002, vol. 20, p. 393-414.

SILVA SANTAMARÍA, Liliana Patricia, et al. Evaluación de agroecosistemas mediante indicadores de sostenibilidad ambiental, económica y social en San José de las Lajas, provincia de Mayabeque, Cuba. 2014.

DE SANTANDER, Alcaldía de Ocaña-Norte. Sitio oficial de Ocaña en Norte de Santander, Colombia. Recuperado de: http://ocana-nortedesantander. gov. co/index. shtml, 2018. 